Review Article

\title{
A REVIEW ON ANTI-INFLAMMATORY POTENTIAL OF SUBSTITUTED PYRAZOLINE DERIVATIVES SYNTHESISED FROM CHALCONES
}

\author{
MURALIDHARAN V., ASHA DEEPTI C. ${ }^{*}$, RAJA S. \\ Department of Pharmaceutical Chemistry, GITAM Institute of Pharmacy, GITAM (Deemed to be University), Visakhapatnam 530045, \\ Andhra Pradesh, India \\ Email: ashadeepti.choppala@gitam.edu
}

Received: 20 Nov 2017 Revised and Accepted: 03 Jan 2018

\begin{abstract}
The pyrazoline ring is a ubiquitous structural feature of many natural and synthetic compounds with potent anti-inflammatory activity. The creation of novel pyrazoline derivatives and examination of their chemical and biological behaviour have gained additional focus in the current decade. Pyrazolines and its fused heterocyclic derivatives tested with anti-inflammatory activity constitute a significant class of compounds for novel drug evolution. Pyrazoline nucleus when linked with different substituents like alkyl, aromatic, heterocyclic rings and many other groups at different positions on the ring shows considerable to more effective anti-inflammatory activity. This article presents a comprehensive review of the anti-inflammatory activity of some novel derivatives of pyrazoline ring.
\end{abstract}

Keywords: Pyrazolines, Chalcones, Phenylhydrazine, Thiosemicarbazide, Anti-inflammatory activity, Carrageenan and Cyclooxygenase

(c) 2018 The Authors. Published by Innovare Academic Sciences Pvt Ltd. This is an open-access article under the CC BY license (http://creativecommons.org/licenses/by/4.0/) DOI: http://dx.doi.org/10.22159/ijpps.2018v10i2.23772

\section{INTRODUCTION}

Heterocyclic compounds play a vital role in all the aspects of biological activities of life. Many biologically active compounds like amino acids, vitamins, hormones, nucleic acids, alkaloids, dyes, drugs (natural and synthetic) possess heterocyclic ring moieties in their structural nucleus. A great deal of research is being done from time to time on heterocyclic compounds in the synthesis of new potent pharmacologically active compounds. Pyrazoline is one such important heterocyclic compound having two nitrogen atoms adjacent to each other and three carbon atoms in a five member ring. The pyrazoline ring is a ubiquitous structural feature of many natural and synthetic compounds with diversified pharmacological efficacy. Its activity gets enhanced when substituted with different aliphatic and aromatic groups at different positions on the ring. Depending upon the substituted group on pyrazole ring, it shows a number of pharmacological activities like antimicrobial [1], anti-inflammatory [2], antioxidant [3], analgesic [4], anti-convulsant [5], anticancer [6], antimalarial [7], anti-Alzheimer's [8] and many more. Hence tremendous research is currently underway to synthesize fruitful pyrazoline derivatives in the field of medicinal chemistry. The first pyrazoline derivative used in the treatment of pain and inflammation is antipyrine (2,3-dimethyl-1-phenyl-3-pyrazolin-5-one). Many drugs are available commercially in pyrazoline nucleus with potent COX2 inhibitor. Some examples of pyrazoline derivatives as NSAIDs are muzolimine and ramifenazone. Phenylbutazone acts as a non steroidal anti-inflammatory drug. The following review furnishes the advances made in the synthetic procedures and the antiinflammatory activity of pyrazolines.

\section{Anti-inflammatory activity of pyrazoline derivatives}

\section{Pyrazolines linked with propargylated phenyl ring}

Kumar et al. [9] reported the synthesis of novel o-propargylated$\mathrm{N}$-acetylpyrazole analogs 1 (fig. 1) by treating 1-acetyl-3,5-diaryl4,5-dihydro(1H)pyrazoles derived from 1,3-diaryl propenones with propargyl bromide. Both o-propargylated-N-acetylpyrazole analogs and $\mathrm{N}$-acetylpyrazole analogs were evaluated for antiinflammatory activity using the carrageenan-induced paw edema method in rats. The derivatives having bromo and nitro groups on the substituted phenyl ring showed comparable activity to that of standard drug.

\section{Pyrazolines linked with $\mathrm{N}$-acyl alkoxy side chain}

Abbas and Naseer [10] reported the synthesis of new pyrazoline derivatives 2 and 3 (fig. 1) equipped with $\mathrm{N}$-acyl arms and homologous alkyloxy side chains which are characterized on the basis of spectroscopic data and microanalysis. The derivatives were screened for in vitro anti-inflammatory activity to examine the effect of alkyloxy side chain length on activity. It was observed that the derivatives which are having the odd number of carbons in the alkyloxy side chain showed better activity. The derivatives with methyoxy and propoxy side chain on phenyl ring present at position5 of pyrazoline ring showed better activity when compared to standard drug indomethacin.

\section{Pyrazolines linked with p-amino benzene sulphonyl group}

Awati et al. [11] reported the synthesis of novel substituted 1,2 pyrazoline derivatives 4 (fig. 1) from chalcones upon reacting with hydrazine hydrate in dry benzene. The synthesized pyrazolines were treated with p-acetamido benzene sulphonyl chloride $(1: 1)$ in benzene to yield p-amino benzene sulphonyl 1,2-pyrazoline 5 (fig. 1). Both the derivatives were evaluated for anti-inflammatory activity by using carrageenan-induced rat hind paw edema. The modifications of synthesized pyrazolines showed remarkable anti-inflammatory activity.

\section{Pyrazolines linked with cycloalkyl group}

Khalil et al. [12] reported a series of novel 5-aryl-3-cyclopropyl-4,5dihydropyrazole derivatives 6 (fig. 1) synthesized via cyclization of chalcones with thiosemicarbazide or semicarbazide $\mathrm{HCl}$ and evaluated for anti-inflammatory activity. The compound 5-(4-chlorophenyl)-3cyclopropyl-4,5-dihydro-1H-pyrazole-1-carbothioamide showed efficient anti-inflammatory activity compared to that of standard drug.

\section{Pyrazolines linked with galloyl group}

Triacetylgalloyl pyrazoline derivatives were afforded by treating the pyrazolines derived from respective chalcones with gallic acid chloride in the presence of triethylamine (TEA) in $\mathrm{CH}_{2} \mathrm{Cl}_{2}$. Triacetylgalloyl pyrazoline derivatives upon treatment with hydrazine monohydrate in $\mathrm{CH}_{3} \mathrm{CN}$ afforded the desired galloyl pyrazoline derivatives 7 (fig. 1) and was reported by Abdel-Aziz M. and GamalEldeen AM. [13]. All the synthesized derivatives were screened for 
anti-inflammatory activity through measurement of the accumulation of nitrites using Griess reagent. The galloyl pyrazoline derivatives with
2,6 dichloro phenyl and 3-hydroxyphenyl substituents at position-5 on pyrazoline ring showed the potent anti-inflammatory activity.<smiles>[R]c1cc(C2=NN(C(C)=O)C(c3ccc(OC=C)c(OC)c3)C2)cc([R3])c1[R]</smiles><smiles>[R]Oc1ccc(C2CC(c3ccccc3)=NN2C(C)=O)cc1</smiles><smiles>[R]c1ccc(C2=NN(O[Na])C(c3ccc([R])c([R])c3[R])C2)cc1</smiles><smiles>[R]C1CC(Nc2ccc(O)cc2)=NN1</smiles>

8<smiles>[X]C(N)N1N=C(C2CC2)CC1c1ccccc1</smiles><smiles>[R]C1CC(N=NC(C)=O)C(c2cn(-c3cccc(Cl)c3)nc2-c2ccc(OC)cc2)C1</smiles>

9<smiles>[R]c1ccc(-c2nn(-c3ccccc3)cc2C2CC(c3ccc(F)cc3)=NN2C(C)=O)cc1</smiles>

11

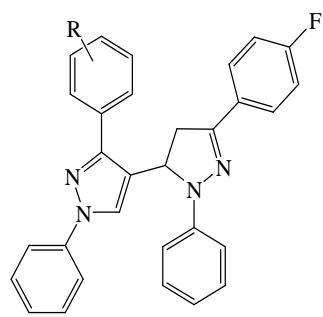

12

Fig. 1: Structures of anti-inflammatory active pyrazoline derivatives 1-12

\section{Pyrazolines linked with aminophenol}

Sahu et al., [14] reported a series of novel 4-(5-substituted aryl-4,5dihydropyrazole-3-yl-amino) phenols 8 (fig. 1) synthesized by treating substituted aryl-N-chalconyl aminophenols with hydrazine hydrate and investigated for anti-inflammatory activity. The observed increase in anti-inflammatory activity is attributed due to the presence of $4-\mathrm{NO}_{2}, 2-\mathrm{OH}$ and $4-\mathrm{Cl}$ phenyl ring at position-5 on pyrazoline ring of synthesized compounds.

\section{Pyrazolines linked with pyrazole moiety}

Nossier et al. [15] reported the synthesis of a new series of substituted pyrazoline derivatives 9 (fig. 1) and 1-thiocarbomoyl pyrazoline derivatives 10 (fig. 1) with nitrogenous heterocyclic ring systems at the C-4 position, from (E)-1-(4-substituted phenyl)-3-(1(3-chlorophenyl)-3-(4-methoxyphenyl)-1H-pyrazol-4-yl) prop-2-en1-one through different chemical reactions and validated by means of spectral and elemental data. The newly synthesized derivatives were evaluated for anti-inflammatory activity using the carrageenan-induced paw edema standard technique. The compound 1-(3-(4-Bromo-phenyl)-5-(1-(3-chloro-phenyl)-3- (4-methoxy-phenyl)-
1H-pyrazol-4-yl)-4,5-dihydro-pyrazol-1-yl ethanone showed consistently excellent anti-inflammatory activity. Jadhav et al. [16] reported the synthesis of a new series of fluoro-substituted pyrazoline derivatives 11 and 12 (fig. 1) from the corresponding pyrazole chalcones in excellent yield by using polyethylene glycol400 as the medium and evaluated for anti-inflammatory activity by using diclofenac drug as standard. The derivatives having phenyl substituted pyrazole on the 5-position of pyrazoline ring showed more potent activity than the standard drug.

\section{Pyrazolines linked with indole ring}

Minakshi Shro and Daharwal [17] reported novel series of indolyl pyrazolines 13 (fig. 2) synthesized by the reaction of chalcones and phenylhydrazine hydrate. The 3-indolaldehyde prepared using Vielsmeier Haack method was utilized for the synthesis of chalcones by reacting with selected aryl ketone. The synthesized pyrazoline derivatives were evaluated for anti-inflammatory activity using carrageenan induced paw edema method in presence of indomethacin as standard drug. It was observed that the unsubstituted N1 of these indolyl pyrazolines were found to give better activity than other N1-substituted indolyl pyrazolines. 


\section{Pyrazolines linked with imidazole ring}

Kishore et al. [18] reported the synthesis of six new derivatives of pyrazolyl imidazo [1,2-a] pyridines 14 (fig. 2). Different phenacyl bromides were treated with 2-aminopyridine to give 2-aryl imidazo [1,2a] pyridines, which on subsequent reaction with Vilsmeier-Haack reagent gave 2-aryl imidazo[1,2-a] pyridine carbaldehydes. From this 2aryl imidazo [1,2-a] pyridine carbaldehydes, chalcones were synthesized by treating with differently substituted acetophenones. Finally, the synthesized chalcones were treated with phenylhydrazines to yield the desired compound. The compounds were evaluated for antiinflammatory activity by carrageenan-induced paw edema method. The presence of chloro and methyl substituted phenyl rings on pyrazoline at different positions showed potent activity when compared to that of standard drug ibuprofen.

\section{Pyrazolines linked with phenyl quinazolinone}

Nallasivan et al. [19] reported the synthesis of eight novel derivatives of 3-[4-(1-acetyl-5-(3,4-disubstitutedphenyl)-4,5dihydro-1H-pyrazol-3-yl)phenyl]-2-substituted phenylquinolin4(3H)-one 15 (fig. 2) by condensation between substituted quinazolinones and hydrazine hydrate in the presence of glacial acetic acid. All the derivatives were evaluated for anti-inflammatory activity by paw edema method using indomethacin as a reference standard. The derivatives having an electron withdrawing groups like nitro and chloro substituents at the para position of phenyl ring on pyrazoline exhibited good anti-inflammatory activity. Rest of the derivatives showed moderate anti-inflammatory activity when compared to that of standard drug.

\section{Pyrazolines linked with acridine ring}

Chandra et al. [20] reported a series of 1-(2',4'-disubstituted acridin9'-yl)-3-(5'-pyridin-4-yl)-(1,3,4oxadizol-2-yl-thiomethyl)-pyrazol-5one 16 (fig. 2) and evaluated for anti-inflammatory activity by using carrageenan induced paw edema method. The compound 1- $\left(2^{\prime}, 4^{\prime}-\right.$ Chloroacridine-9'-yl)-3-(5'-pyridine-4-yl)-(1,3,4-oxadiazol-2-ylthiomethyl)-pyrazole-5-one showed better anti-inflammatory activity at the three graded dose of 25,50 and $100 \mathrm{mg} / \mathrm{kg} \mathrm{p}$. o. due to the presence of electron withdrawing chloro group at 2 and 4 positions of acridine ring.

\section{Pyrazolines linked with coumarine ring}

Khode et al. [21] reported the synthesis of novel series of 5(substituted) aryl-3-(3-coumarinyl)-1-phenyl-2-pyrazolines 17 (fig. 2) from substituted 3-aryl-1-(3-coumarinyl) propan-1-ones reacting with phenylhydrazine in the presence of hot pyridine. The synthesized compounds were evaluated for the anti-inflammatory activity. Compounds with chloro, dichloro, methoxy and fluoro substituted phenyl ring at position- 5 on pyrazoline ring exhibited significant anti-inflammatory activity in model of acute inflammation such as carrageenan-induced rat paw edema while compounds chloro, dichloro substituted phenyl ring at position- 5 on pyrazoline ring showed considerable activity in model of chronic inflammation such as adjuvant-induced arthritis and were compared with diclofenac as a standard drug.

\section{Pyrazolines linked with morpholinophenyl group}

Joshi et al. [22] designed and reported a series of 3,2-(4,5-dihydro-5(4-morphilinophenyl)-1H-pyarazol-3-yl)phenols 18 (fig. 2) and its $\mathrm{N}$-phenylpyrazol-1-carbothioamide 19 (fig. 2). The chalcone (E)-1(2-Hydroxyphenyl)-3-(4-morpholinophenyl) prop-2-en-1-one was synthesized by Claisen-Schmidt condensation of 4-(morpholin-4-yl) benzaldehyde with substituted 2-hydroxyacetophenones in a dilute ethanolic solution of potassium hydroxide at ambient temperature. These chalcones upon treating with hydrazine hydrate yield the desired pyrazolines. All the derivatives were evaluated for antiinflammatory activity. It was found that the presence of bromo and chloro groups on hydroxy phenyl at position-5 of pyrazoline ring showed good anti-inflammatory activity.

\section{Pyrazolines linked with benzofuran moiety}

New series of seven 2-[5'-phenyl(4" ${ }^{\prime \prime}$-oxyacetic acid)-1-aryl ketano pyrazoline)]-7-methoxy benzofuran 20 (fig. 2) were synthesized and reported for their anti-inflammatory activity by using carrageenaninduced rat hind paw oedema method by Suthakaran et al. [23]. The derivatives having a p-fluorophenyl ring and 2-amino-5-bromo phenyl ring at the N1 position of pyrazoline showed comparatively good anti-inflammatory activity with that of standard drug.

\section{Pyrazolines linked with thiophene substitution}

Reddy et al. [24] synthesized novel pyrazolines 21 (fig. 2) from the chalcones obtained by condensing different aldehydes with 2-acetyl5-bromothiophene and evaluated for anti-inflammatory activity by the in vitro methods like inhibition of bovine albumin denaturation and heat-induced hemolysis methods. It was found that the compounds with chloro substitution have moderate in vitro antiinflammatory activity. Jainey and Bhat [25] reported the synthesis of 5-(substituted phenyl)-1-phenyl-3-(thiophen-2-yl)-4, 5-dihydro-1Hpyrazoles 22 (fig. 2) and 5-(substituted phenyl)-3-(thiophen-2-yl)4,5-dihydro-1H-pyrazoles 23 (fig. 2) from intermediate chalcones which are prepared by condensing 2 -acetyl thiophene with various substituted benzaldehydes in the presence of $\mathrm{NaOH}$. This chalcone on cyclization with phenylhydrazine and hydrazine hydrate in presence of pyridine as catalyst gave pyrazolines. The synthesized pyrazolines were evaluated for anti-inflammatory activity using the carrageenan-induced rat paw edema inhibition method in presence of indomethacin as standard drug. It was observed that the synthesized compounds with electron withdrawing groups like halogens on the aromatic ring enhance the anti-inflammatory activity. Ramesh and Sumana [26] reported the synthesis of new pyrazoline derivatives 24 (fig. 2). Initially, chalcones were synthesized by condensing 2-acetyl thiophene with different aromatic aldehydes. This chalcone upon treated with phenylhydrazine hydrochloride in the presence of alcohol yields pyrazolines and were screened for anti-inflammatory activity by using carrageenan induced paw edema method. It was observed that the derivatives having chlorine and fluorine-substituted phenyl ring at position-3 on pyrazoline ring showed maximum antiinflammatory activity when compared to the standard drug aceclofenac.

\section{Pyrazolines linked with indole}

El-Sayed et al. [27] synthesized and reported pyrazoline derivatives 25 (fig. 3) and their ability to inhibit ovine COX-1/COX-2 isozymes was evaluated using in vitro cyclooxygenase (COX) inhibition assay. First chalcones were prepared by crossed aldol condensation between isatin and acetophenone derivatives in the presence of dimethylamine as a basic catalyst followed by dehydration. Nucleophilic Michael addition of hydrazine hydrate to the reaction mixture yields spiropyrazoline via a one-pot synthesis without the isolation of the intermediate chalcones. It was found that the synthesized compounds exhibit optimal COX-2 inhibitory potency IC50 $=0.26 \mathrm{IM}$ and selectivity index (SI) $=>192.3$ comparable with reference drug celecoxib (IC50 value of $0.28 \mathrm{IM}$ and selectivity index of 178.57).

\section{Pyrazolines linked with halobenzene}

Khalil and Refaat [28] reported the synthesis of eleven new 3-(4bromophenyl)-5-(4-fluorophenyl)-2-pyrazoline derivatives 26 (fig. 3) by condensing 1-(4-bromophenyl)-3-(4-fluorophenyl) prop-2en-1-one and phenylhydrazine. The synthesized compounds were evaluated for anti-inflammatory activity by using carrageenaninduced edema in albino rats at a dose of $10 \mathrm{mg} / \mathrm{kg}$. It was found that presence of $\mathrm{N}-1$ formyl, thiocarbamoyl, propyl or 3-(thiophen2-yl)-2-propen-1-yl-1-one as a substituent on pyrazoline ring elicit a remarkable increase in anti-inflammatory activity. Bano et al. [29] reported the synthesis of some new 2-pyrazolines 27 (fig. 3) bearing benzenesulfonamide moiety by reacting chalcones/ flavanones with 4-hydrazinonbenzenesulfonamide hydrochloride. The synthesized compounds were tested for anti-inflammatory activity by carrageenan-induced rat paw edema bioassay and found safe from the point of view of ulcer induction. The compounds having 4-chloro phenyl ring and 2-hydroxyphenyl ring at position-3 on pyrazoline ring showed maximum activity and showed minimum inhibition against the enzymatic activity of COX1 and COX-2. 

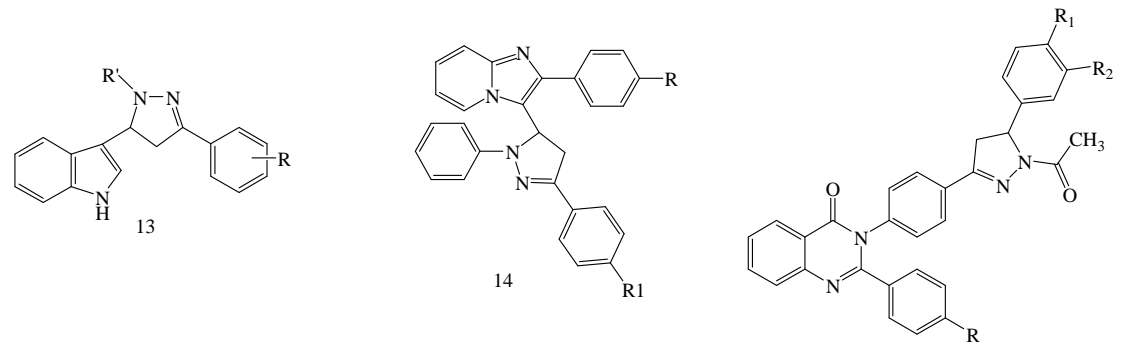

15

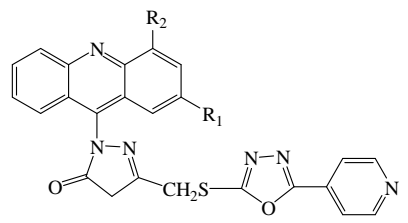

16
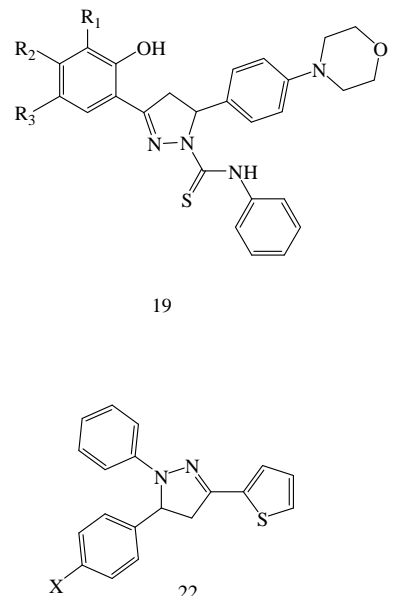<smiles>CC1CC(C2C(O)C(=O)C3CCCCC32)NC1C1CCCCC1</smiles>

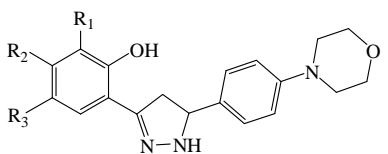

18<smiles>CC(=O)N1N=C(c2ccc(Br)s2)CC1[Al]</smiles>
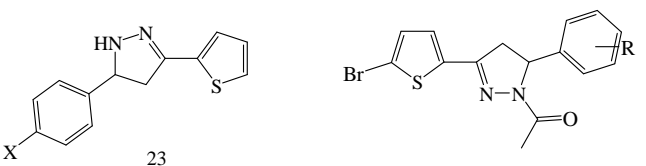

24

Fig. 2: Structures of anti-inflammatory active pyrazoline derivatives 13-24

\section{Pyrazolines linked with sulphonamide}

Rahul et al. [30] designed and reported novel 1-(4-methylsulphonyl amino methyl)phenyl-3,5-diaryl-pyrazolines 28 (fig. 3) bearing aryl and sulphanoamido pharmacophores by the convenient synthetic protocol as cyclocondensation of 1,3-diaryl 2-propene-1-ones and 4(sulphonylamino methyl)-phenyl hydrazine hydrochloride in ethanol and triethanolamine. The synthesized compounds were evaluated for anti-inflammatory activity by HRBC membrane stabilization method. The derivatives having 4-methoxyphenyl and 4-fluorophenyl groups at position-5 of pyrazoline ring showed most potential activity. A new series of pyrazolyl urea 29 (fig. 3) and pyrazolyl sulphonamide 30 (fig 3.) were synthesized and reported by Amir et al. [31], from the starting materials phenyl isocyanate and p-acetamido benzenesulphonyl chloride. All the compounds were screened for anti-inflammatory activity. The compounds 1-(4-(1acetyl-5-substituted-4,5-dihydro-1 $H$-pyrazole-3yl)phenyl)-3-phenyl urea derivatives having substitutions of chloro, nitro and anthracinyl groups and 4-acetamido-N-(4-(1-acetyl-5-substituted-4,5-dihydro$1 H$-pyrazole-3yl)phenyl)-3-phenyl sulphonamide derivatives having chloro and anthracinyl substituted groups showed significant antiinflammatory activity. Bashir et al., [32] reported the synthesis of thirteen new 2-pyrazoline derivatives 31 (fig. 3) bearing benzenesulfonamide moiety by condensing appropriate chalcones with 4-hydrazinonbenzene sulfonamide hydrochloride. The derivatives having 3,4,5-trimethoxy phenyl and N,N-dimethylamino phenyl groups showed potent activity when compared to that of standard drug. Rathish et al. [33] designed and reported new 2- pyrazoline bearing benzenesulfonamide derivatives 32 (fig. 3) by condensing chalcones with 4-hydrazinon benzene sulfonamide hydrochloride. These compounds were tested for their antiinflammatory activity by carrageenan-induced rat paw edema model at a dose of $20 \mathrm{mg} / \mathrm{kg}$ and volume of paw edema was measured at 0 , 3 and $5 \mathrm{~h}$. The compound with methoxy groups and dimethylamino groups showed good activity.

\section{Pyrazolines linked with methasulfonyl phenyl ring}

Fioravanti et al. [34] reported a series of methansulfonyl phenyl substituted pyrazoline derivatives 33 (fig. 3) and evaluated them for anti-inflammatory activity. A solution of appropriate 4-methyl sulfonyl chalcone and hydrazine hydrate or thiosemicarbazide in $50 \mathrm{ml}$ of ethanol and sodium hydroxide was refluxed for $8 \mathrm{~h}$ in presence of acetic acid to yield the pyrazoline derivatives. The synthesized derivatives with methyl and methoxy-substituted phenyl ring at position-5 of pyrazoline exhibited more potent anti-inflammatory activity.

\section{Pyrazolines linked with carbothioic acid}

Harathi et al. [35] reported the synthesis of substituted 3,5diphenyl-4,5-dihydropyrazole-1-carbothioic acid benzylideneamide 34 (fig. 3) from chalcones and evaluated for anti-inflammatory activity by using egg-albumin induced paw edema method. The synthesized pyrazole derivatives having alkenes and electron withdrawing chloro and nitro groups showed potent antiinflammatory activity when compared to that of standard drug diclofenac sodium. 

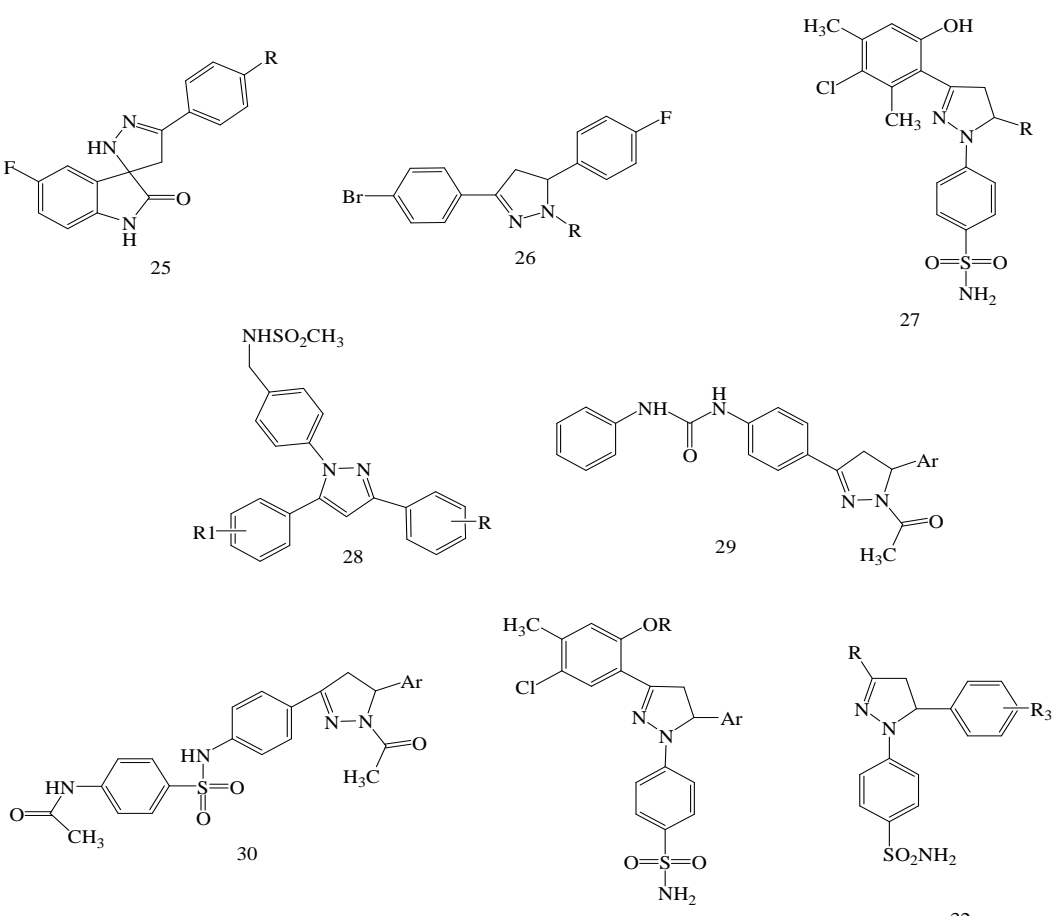

31
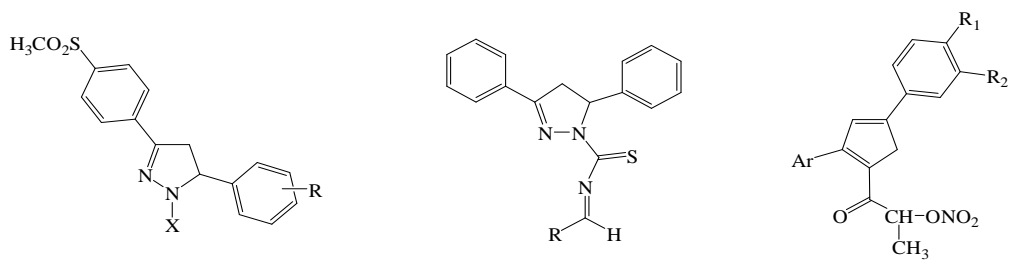

33

Fig. 3: Structures of anti-inflammatory active pyrazoline derivatives 25-35

\section{Pyrazoline linked with nitric oxide donating group}

Shoman et al., [36] reported the synthesis of novel nitric oxidedonating pyrazoline derivatives 35 (fig. 3 ) by carrying a nitrate ester group or an oxime group onto the prepared 3,5-diaryl-2-pyrazoline derivatives through different spacers and were evaluated for their anti-inflammatory activity using carrageenan-induced rat paw edema and compared with indomethacin as a reference drug. It was observed that the incorporation of the NO-donating group into the parent pyrazoline derivatives caused a non-significant reduction in the anti-inflammatory activity.

\section{CONCLUSION}

The plethora of research elucidated in this review furnishes the antiinflammatory activity of pyrazoline derivatives. A further modification in its main nucleus provides more efficient derivatives with more potent therapeutic efficacy. This review illustrates many efficient protocols for the synthesis and evaluation of antiinflammatory activity of pyrazoline nucleus substituted with different aromatic, heterocyclic and other groups.

\section{ACKNOWLEDGEMENT}

The authors are thankful to the UGC (New Delhi, India) for providing financial assistance to GITAM Institute of Pharmacy, Gandhi Institute of Technology And Management (Deemed to be University), Visakhapatnam, Andhra Pradesh, India.

\section{AUTHORS CONTRIBUTIONS}

The complete review work was suggested and designed by Raja $\mathrm{S}$ and Asha Deepti C. Literature collection and written works were carried out by Muralidharan V. The review manuscript was drafted by Muralidharan V. The manuscript was edited by Raja S and Asha Deepti C. Authors read and approved the final manuscript.

\section{CONFLICT OF INTERESTS}

Authors declare no conflict of interest

\section{REFERENCES}

1. Ozdemir A, Turan Zitouni G, Kaplancıklı ZA, Revial G, Guven K Synthesis and antimicrobial activity of 1-(4-aryl-2-thiazolyl)-3(2-thienyl)-5-aryl-2-pyrazoline derivatives. Eur J Med Chem 2007:42:403-9.

2. Fernandes J, Revanasiddappa BC, Ishwarbhat K, Kumar MV, D'Souza L, Alva SS. Synthesis and in the vitro anti-inflammatory activity of novel pyrazoline derivatives. Res J Pharm Technol 2017;10:1679-82.

3. Kumar A, Varadaraj BG, Singla RK. Synthesis and evaluation of antioxidant activity of novel 3,5-disubstituted-2-pyrazolines. Bull Fac Pharm Cairo Univ 2013;51:167-73.

4. Sridhar S, Rajendraprasad Y. Synthesis and analgesic studies of some New 2-pyrazolines. J Chem 2012;9:1810-5.

5. Beyhan N, Kaymakcioglu BK, Gumru S, Aricioglu F. Synthesis and anticonvulsant activity of some 2-pyrazolines derived from chalcones. Arabian J Chem 2017;10 Suppl 2:S2073-81.

6. Montoya A, Quiroga J, Abonia R, Nogueras M, Cobo J, Insuasty B. Synthesis and in vitro antitumor activity of a novel series of 2 pyrazoline derivatives bearing the 4-aryloxy-7-chloroquinoline fragment. Molecules 2014;19:18656-75.

7. Akhtar W, Khan MF, Verma G, Shaquiquzzaman M, Akhter M, Marella A, et al., Coumarin-pyrazoline derivatives: their onepot microwave assisted synthesis and antimalarial activity. J Med Chem 2017;3:5-9. 
8. Upadhyay S, Tripathi AC, Paliwal S, Saraf SK. 2-pyrazoline derivatives in neuropharmacology: synthesis, ADME prediction, molecular docking and in vivo biological evaluation. EXCLI J 2017;16:628-49.

9. Kumar DA, Bhawna C, Rameshwar D, Sanjeev KM. Synthesis and anti-inflammatory activity of some o-propargylated-Nphenylpyrazole derived from 1,3-diarylpropenones. Int J Med Chem 2016;1-6. Doi:10.1155/2016/3156593.

10. Abbas A, Naseer MM. Synthesis and anti-inflammatory activity of new N-acyl-2-pyrazolines bearing homologous alkyloxy side chains. Acta Chim Slov 2014;61:792-802.

11. Gonjare NS, Awati SS, Sachin RK, Pramod BP, Shitalkumar SP, Manish SK. Synthesis and anti-inflammatory activity of some novel 1,2-pyrazoline derivatives. Pharma Chem 2013;5:86-91.

12. Khalil NA, Ahmed EM, El-Nassan HB, Ahmed OK, Al-Abd AM. Synthesis and biological evaluation of novel pyrazoline derivatives as anti-inflammatory and antioxidant agents. Arch Pharm Res 2012;35:995-1002.

13. Abdel Aziz M, Gamal Eldeen AM. Synthesis and screening of anticancer, antioxidant, and anti-inflammatory activities of novel galloyl pyrazoline derivatives. Pharm Biol 2009;47:854-63.

14. Sahu SK, Banerjee M, Samantray A, Behera C, Azam MA. Synthesis, analgesic, anti-inflammatory and antimicrobial activities of some novel pyrazoline derivatives. Trop J Pharm Res 2008;7:961-8.

15. Nossier ES, Fahmy HH, Khalifa NM, El-Eraky WI, Baset MA Design and synthesis of novel pyrazole-substituted different nitrogenous heterocyclic ring systems as potential antiinflammatory agents. Molecules 2017;22:512, 1-16.

16. Shravan YJ, Sachin PS, Suresh DK, Sandeep BP, Sharad KP, Raghunath BB. PEG-mediated synthesis and pharmacological evaluation of some fluoro substituted pyrazoline derivatives as anti-inflammatory and analgesic agents. Bioorg Med Chem Lett 2013;23:2575-8

17. Shroff M, Daharwal SJ. Newer substituted indolyl-pyrazoline derivatives as anti-inflammatory agents. $\mathrm{Br}$ Biomed Bull 2017;5:298.

18. Kishore BN, Rishika U, Ameena B, Hepsibha C, Madhava Reddy $\mathrm{B}$, Babu VH. Synthesis, characterization of some novel pyrazoline incorporated imidazo [1,2-a]pyridines for antiinflammatory and antibacterial activities. Pharma Chem 2017;9:45-9.

19. Kumar Nallasivan P, Jayakar B, Gopal N. Anti-inflammatory activity of novel pyrazoline containing quinazolinone derivatives. Asian J Pharm Anal Med Chem 2015;3:31-8.

20. Chandra T, Neha G, Suman L, Saxena KK, Ashok Kumar. Synthesis of substituted acridinyl pyrazoline derivatives and their evaluation for anti-inflammatory activity. Eur J Med Chem 2010;45:1772-6.

21. Khode S, Maddi V, Aragade P, Palkar M, Ronad PK, Mamledesai $\mathrm{S}$, et al. Synthesis and pharmacological evaluation of a novel series of 5-(substituted)aryl-3-(3-coumarinyl)-1-phenyl-2pyrazolines as novel anti-inflammatory and analgesic agents. Eur J Med Chem 2009;44:1682-8.

22. Joshi RS, Priyanka GM, Santosh DD, Sanjay KD, Charansingh HG. Synthesis, analgesic and anti-inflammatory activities of some novel pyrazolines derivatives. Bioorg Med Chem Lett 2010; 20:3721-5.

23. Suthakaran R, Somasekhar G, Sridevi CH, Marikannan M, Suganthi K, Nagarajan G. Synthesis, anti-inflammatory, antioxidant and antibacterial activities of 7-methoxy benzofuran pyrazoline derivatives. Asian J Chem 2007;19: 3353-62.

24. Siva Sanker Reddy L, Bhagavan Raju M, Sridhar C. Novel pyrazolines: synthesis and evaluation of their derivatives with anticancer and anti-inflammatory activities. Int J Pharm Pharm Sci 2015;8:247-54.

25. Jainey PJ, Bhat IK. Antitumor, analgesic, and anti-inflammatory activities of synthesized pyrazolines. J Young Pharm 2012;4:82-7.

26. Ramesh B, Sumana T. Synthesis and anti-inflammatory activity of pyrazolines. J Chem 2010;7:514-6.

27. Magda AA, Abdel-Aziz NI, Alaa AM, El-Azab AS, ElTahir KEH. Synthesis, biological evaluation and molecular modelling study of pyrazole and pyrazoline derivatives as selective COX-2 inhibitors and anti-inflammatory agents. Bioorg Med Chem 2012;20:3306-16.

28. Khalil OM, Refaat HM. Synthesis and anti-inflammatory activity of some 3, 5-diaryl-2-pyrazoline derivatives. Orient J Chem 2011;27:1581-90.

29. Bano S, Kalim J, Shamim A, Rathish IG, Surender S, Alam MS. Synthesis and biological evaluation of some new 2-pyrazolines bearing benzenesulfonamide moiety as potential antiinflammatory and anti-cancer agents. Eur J Med Chem 2011;46:5763-8.

30. Rahul AW, Dinesh LL, Vrushali P, Ashish A. Synthesis and in vitro anti-inflammatory activity of some 1-(4-methylsulphonyl aminomethyl) phenyl-3, 5-diaryl-pyrazolines. Int J ChemTech Res 2017;10:201-6.

31. Amir M, Ali S, Somakala K. Synthesis and biological evaluation of some new pyrazoline derivatives as anti-inflammatory agents. Indian J Chem 2016;55B:478-85.

32. Bashir R, Ovais S, Yaseen S, Hamid H, Alam MS, Samim M, et al. Synthesis of some new 1,3,5-trisubstituted pyrazolines bearing benzene sulfonamide as anticancer and anti-inflammatory agents. Bioorg Med Chem Lett 2011;21:4301-5.

33. Rathish IG, Javed K, Ahmad S, Bano S, Alam MS, Pillai KK, et al. Synthesis and anti-inflammatory activity of some new 1,3,5trisubstituted pyrazolines bearing benzene sulphonamide. Bioorg Med Chem Lett 2009;19:255-8.

34. Fioravanti R, Adriana B, Fedele M, Francesca R, Francisco O, Francesco 0 , et al. Synthesis and biological evaluation of $\mathrm{N}$ substituted-3,5-diphenyl-2-pyrazoline derivatives as cyclooxygenase (COX-2) inhibitors. Eur J Med Chem 2010; 45:6135-8.

35. Harathi P, Rajendra Prasad VVS, Satyavati D, Subramanian S, Vivekananda B, Prashanthi G. Synthesis, characterization and anti-inflammatory activity of novel pyrazole derivatives. Asian J Pharm Clin Res 2015;8:82-6.

36. Shoman ME, Abdel Aziz M, Omar MA, Hassan HF, Morsy MA. Synthesis and investigation of anti-inflammatory activity and gastric ulcerogenicity of novel nitric oxide-donating pyrazoline derivatives. Eur J Med Chem 2009;44:3068-76. 